Psychotherapeut 2014 $59: 81-81$

DOI 10.1007/s00278-014-1035-7

Online publiziert: 19. Februar 2014

(c) Springer-Verlag Berlin Heidelberg 2014

Manfred Cierpka ${ }^{1}$. Verena Kast ${ }^{2}$ - Peter Henningsen ${ }^{3}$

${ }^{1}$ Institut für Psychosomatische Kooperationsforschung und Familientherapie, Psychosoziales Zentrum, Uniklinikum Heidelberg

${ }^{2}$ C.G.-Jung-Institut, Zürich, Schweiz

${ }^{3}$ Klinik für Psychosomatische Medizin und Psychotherapie, Klinikum rechts der Isar,

Technische Universität München

\title{
Herausforderungen an die Psychotherapie heute
}

Schweitzer-Rothers postuliert in sei-

Wir alle, aber im besonderen Maß unsere Patienten, klagen über die zunehmende Beschleunigung im digitalen Zeitalter. Immer mehr soll in immer kürzer werdenden Zeitspannen geleistet werden. Die persönlichen Ressourcen werden durch diese Anforderungen häufig ausgereizt. Die stärkere Vernetzung der Menschen, die durch die digitalen Kommunikationswege möglich wurde, ist überall zu beobachten und trägt ebenfalls zu rasanten $\mathrm{Be}$ schleunigungen in unserem privaten und beruflichen Leben bei. Nicht alle sind für diese Herausforderungen gerüstet. Gerade die vulnerablen Menschen spüren den Druck am deutlichsten. Die psychotherapeutische Profession wird, ob sie will oder nicht, zur „Reparationskultur“ für diese neue Welt.

Die gesellschaftlichen Veränderungen führen zu „neuen Ängsten“ bei den Patienten, mit denen wir in der Psychotherapie konfrontiert werden.

Gibt es in der Praxeologie der Psychotherapie darauf eine Antwort? Findet man dort auch „neue Kulturen“, um diesen Herausforderungen zu begegnen? Gibt es gar in der Psychotherapie einen entsprechenden Kulturwandel?

Diese Frage stellten wir uns während der „Lindauer Psychotherapiewochen 2013“ im Rahmen des Leitthemas „Neue Kulturen schaffen“. Alle Referenten der Vortragsreihe "Kulturwandel in der Psychotherapie "überarbeiteten ihre Vortragstexte zu Veröffentlichungen, die in diesem Schwerpunktheft der Zeitschrift Psychotherapeut erscheinen. nem systemischen Beitrag, dass die Entwicklung einer psychischen Erkrankung genauso eine Gemeinschaftsleistung darstellt wie ihre Heilung. Er begründet damit den aktuellen Trend zu häufigeren Mehrpersonentherapien. Er berichtet über neue Therapieformen, die diese Mehrpersonenperspektive berücksichtigen. Vogel weist darauf hin, dass wir heute in der psychotherapeutischen Praxis eine Erweiterung auf existenzielle Themen wie Sinn, Freiheit, Einsamkeit und Tod finden. Eine existenzielle Not kann als Schicksal interpretiert werden. Unsere Patienten fühlen sich in diesen existenziellen Krisen vom Schicksal gebeutelt. Wie können wir darauf antworten? Lambert et al. stellen für die psychiatrisch-psychotherapeutische Versorgung neue integrierte Ansätze zur Behandlung von Psychosen vor. Die Autoren des „Hamburger Modells" fordern einen Wandel in Denken und Handeln in der Psychotherapie von Psychosen, aber auch entsprechende Veränderungen der Versorgungsstrukturen. Da diese Patientengruppe einer Hilfestellung eher misstrauisch gegenübersteht und nur zögernd oder gar unwillig zum Psychotherapeuten kommt, muss die Versorgung um Konzepte ergänzt werden, die die aufsuchende Behandlung in den Mittelpunkt rücken. Resch $u$. Parzer heben in ihrem Beitrag hervor, dass insbesondere bei Jugendlichen eine rein nosologische Sichtweise zu kurz greift, weil jedes Symptom auch in den aktuellen Lebenskontext eingebettet ist und eine entspre- chende Anpassungsleistung darstellt. Die heutige Jugend ist sehr stark mit den Herausforderungen des digitalen Zeitalters konfrontiert. In der Psychotherapie dieser Jugendlichen müssen insofern die funktionellen Verflechtungen der Symptome mit dem Lebenskontext in einem individualisierten Vorgehen in der Therapie berücksichtigt werden. Von Gontard widmet sich dem Thema der Migration in unserer sich wandelnden Gesellschaft. Er konzentriert sich auf die sog. Secondos, Menschen der 2. Generation nach Migration. Die kindliche Sozialisation kann sich je nach Herkunftsland bei diesen Menschen deutlich unterscheiden, was in der Psychotherapie berücksichtigt werden muss. Spezifische kulturelle Erziehungspraktiken und deren Auswirkungen werden beispielhaft anhand von 2 Kasuistiken diskutiert.

Wir bedanken uns bei den Referenten dafür, dass sie ihre Vortragsmanuskripte zu Zeitschriftenbeiträgen überarbeitet haben. Wir wünschen viel Freude bei der Lektüre.

\section{Korrespondenzadresse}

\section{Prof. Dr. Manfred Cierpka}

Institut für Psychosomatische

Kooperationsforschung und Familientherapie,

Psychosoziales Zentrum, Uniklinikum

Heidelberg

Bergheimer Str. 54, 69115 Heidelberg

manfred_cierpka@med.uni-heidelberg.de 